\title{
A Semianalytical Solution of the Fractional Derivative Model and Its Application in Financial Market
}

\author{
Lina Song $\mathbb{D}$ \\ School of Mathematics, Dongbei University of Finance and Economics, Dalian 116025, China \\ Correspondence should be addressed to Lina Song; 1_n_song@163.com
}

Received 25 September 2017; Accepted 28 February 2018; Published 1 April 2018

Academic Editor: Thierry Floquet

Copyright (C) 2018 Lina Song. This is an open access article distributed under the Creative Commons Attribution License, which permits unrestricted use, distribution, and reproduction in any medium, provided the original work is properly cited.

Fractional differential equation has been introduced to the financial theory, which presents new ideas and tools for the theoretical researches and the practical applications. In the work, an approximate semianalytical solution of the time-fractional European option pricing model is derived using the method of combining the enhanced technique of Adomian decomposition method with the finite difference method. And then the result is introduced in China's financial market. The work makes every effort to test the feasibility of the fractional derivative model in the actual financial market.

\section{Introduction}

Since it appeared in 1973, the Black-Scholes model [1, 2] has become the most popular method for option pricing. This classical Black-Scholes equation is a linear parabolic partial differential equation, as follows:

$$
\frac{\partial V}{\partial t}+\frac{1}{2} \sigma^{2} S^{2} \frac{\partial^{2} V}{\partial x^{2}}+r S \frac{\partial V}{\partial S}-r V=0,
$$

where $V=V(S, t)$ is the option price, $S$ is the asset price, $r$ is the risk-free interest rate, and $\sigma$ is the volatility for the asset price $S$.

The Black-Scholes model provides a simple and effective technique to estimate the option price. With the researches developing further, empirical studies [3-6] proved that the financial market has the fractal characters both at home and abroad. So, the classical model established on a partial differential equation with integer-order derivatives is not enough to reflect the reality of the financial market. As everyone knows, the differential equations involving the fractional derivatives are powerful tools to study the fractal geometry and the fractal dynamics. Fractional differential equations show the superiority in modeling the important phenomena in various fields such as fluid flow, electromagnetic, acoustics, electrochemistry, cosmology, and material science. Then, whether the fractional differential equation can be applied into the financial market? The answer is Yes! Fractional derivative has the property of self-similarity and it replies to the long-range dependence better than the derivative with integer order. The superiorities are beneficial to depict the fractal structure in the financial market. In recent years, the researches on the application of fractional calculus in the financial theory show an increasing tendency. Cartea and del-Castillo-Negrete [7] presented the fractional diffusion models of the option prices in the derivatives market. In this article, the authors introduced Riemann-Liouville fractional derivative into the pricing problems of financial derivatives and established the space-fractional diffusion equations using three special processes of FMLS, CGMY, and KoBoL in markets with jumps, which provided new tools to deal with financial problems. Reference [7] made the numerical treatment of knock-out barrier options for FMLS processes and the analysis showed the effectiveness of fractional models. Finally, [7] pointed out that the models can be used to deal with American option. Based on the fractional models presented by Cartea and del-Castillo-Negrete, Marom and Momoniat [8] compared the numerical solutions of three models and analyzed European option. Subsequently, Xi and Cao [9] gave numerical solutions of the fractional American option pricing model by constructing linear complementarity problem. An explicit closed-form analytical solution of the FMLS model was found by Chen et al. [10] where the effectiveness of this model is proved by comparing with derivatives of integer order and the validity of the put-call 
parity is verified. Jumarie $[11,12]$ derived the time and spacetime fractional Black-Scholes equations and gave optimal fractional Merton's portfolio. Based on Jumarie's theoretical derivation, Liang et al. [13] introduced a bifractional BlackScholes model whose time-fractional derivative is produced using the heuristic arguments. Using the heuristic arguments, Chen et al. [14] used a modified Black-Scholes equation with a time-fractional derivative to govern the double barrier option and gave analytical solution. Song [15] presented a space-time fractional derivative model for European option pricing with transaction costs by the replicating portfolio.

The above works present new ideas and methods for the researches on derivatives pricing theory. This study is in the initial stage and lots of problems need to be solved. The aim of the paper is to solve the time-fractional pricing model and test the practicability of the results by the market data. The paper has been organized as follows. In Section 2, the timefractional derivative model of option pricing is succinctly described. In Section 3, the semianalytical solution of the fractional model is derived by the enhanced technique and the finite difference method. In Section 4, the result is tested by the real data from China's warrants and options market. Some problems and conclusions are presented in Section 5.

\section{Time-Fractional Black-Scholes Equation}

Giona and Roman $[16,17]$ pointed out that the relationship between the total flow of the probability flow $i(r, t)$ and the average probability density $p(r, t)$ is

$$
\int_{0}^{t} i(r, \tau) d \tau=r^{d_{f}-1} \int_{0}^{t} K(t-\tau) p(r, \tau) d \tau,
$$

where $d_{f}$ is the dimension of the fractal media. The diffusion kernel of the fractal media can be expressed as

$$
K(t-\tau)=\frac{A_{\alpha}}{\Gamma(1-\alpha)(t-\tau)^{\alpha}}, \quad 0<\alpha<1,
$$

where $\alpha$ is a transmission exponent and $A_{\alpha}$ is a constant that can be determined [18]. Based on [13, 14, 19], $A_{\alpha}$ is supposed as 1 in following work.

The change of option price is considered as a fractal transmission system. Let $V(S, \tau)$ denote the option price with $\tau=T-t$; the relationship between $i(S, \tau)$ and $p(S, \tau)$ for the option problem is determined by (2), as follows:

$$
\int_{0}^{\tau} i\left(S, \tau^{\prime}\right) d \tau^{\prime}=S^{d_{f}-1} \int_{0}^{\tau} K\left(\tau-\tau^{\prime}\right) p\left(S, \tau^{\prime}\right) d \tau^{\prime},
$$

where

$$
\begin{aligned}
& p(S, \tau)=V \\
& i(S, \tau)=\frac{1}{2} \sigma^{2} S^{2} \frac{\partial^{2} V}{\partial S^{2}}+r S \frac{\partial V}{\partial S}-r V .
\end{aligned}
$$

Then, one can establish the following time-fractional model:

$$
S^{d_{f}-1} \frac{\partial^{\alpha} V}{\partial \tau^{\alpha}}=\frac{1}{2} \sigma^{2} S^{2} \frac{\partial^{2} V}{\partial S^{2}}+r S \frac{\partial V}{\partial S}-r V, \quad 0<\alpha<1,
$$

where $\partial^{\alpha} V / \partial \tau^{\alpha}$ is just the Riemann-Liouville fractional derivative defined as

$$
\frac{\partial^{\alpha} V}{\partial \tau^{\alpha}}=\frac{1}{\Gamma(1-\alpha)} \frac{\partial}{\partial \tau} \int_{0}^{\tau} \frac{1}{\left(\tau-\tau^{\prime}\right)^{\alpha}} V\left(S, \tau^{\prime}\right) d \tau^{\prime} .
$$

The idea of the above transformation from a BlackScholes equation with integer-order derivative to a fractional Black-Scholes equation has been adopted for the option problem in $[13,14,19]$. By the relationship presented in the mentioned documents, one can also obtain the timefractional model. The analytical solution of the model containing the dividend yield $D$ and $d_{f}=1$ is given by [14]. $\mathrm{Li}$ [19] gave the numerical solutions of the time-fractional model with $d_{f}$ being 1 by the finite difference method. The main task of this paper is to present solutions of (6) with the condition $d_{f} \neq 1$ and give the actual application. Under the situation, finding analytical solutions is difficult and so it is necessary to turn to semianalytical approximations for getting a solution.

The values of European call and put option in the work are supposed to be a solution of (6) with the following initial and boundary conditions, respectively:

$$
\begin{aligned}
& V(S, 0)=\max (S-K, 0), \\
& V(0, \tau)=0, \\
& V(S, \tau)=S-K \exp (-r \tau), \quad S \longrightarrow+\infty, \\
& V(S, 0)=\max (K-S, 0), \\
& V(0, \tau)=K \exp (-r \tau), \\
& V(S, \tau) \rightarrow 0, \quad S \longrightarrow+\infty .
\end{aligned}
$$

\section{Semianalytical Solution}

In this section, two aspects of work need to be complete. The one is to give an enhanced technique of Adomian decomposition method; the other is to solve a semianalytically solution of the pricing model by the enhanced technique and the finite difference method.

3.1. Description of the Enhanced Method. Consider a general differential equation

$$
[\mathscr{L}+\mathscr{R}+\mathscr{N}] u(x, t)=0,
$$

where $\mathscr{L}$ is an easily or trivially invertible linear operator, $\mathscr{R}$ is the remaining linear part, and $\mathcal{N}$ is a nonlinear operator.

Equation (10) can be rewritten as

$$
u=\Phi-\mathscr{L}^{-1}[\mathscr{R}+\mathcal{N}] u
$$

where $\Phi$ is determined by the given conditions.

The standard Adomian decomposition method [20, 21] suggests the solution $u$ can be decomposed into the infinite series and the nonlinear term $\mathcal{N} u$ is decomposed; namely,

$$
\begin{gathered}
u=\sum_{n=0}^{\infty} u_{n}, \\
\mathcal{N} u=\sum_{n=0}^{\infty} A_{n},
\end{gathered}
$$


where $A_{n}$ are Adomian polynomials, defined by

$$
A_{n}=\frac{1}{n !}\left[\frac{d^{n}}{d \lambda^{n}} \mathscr{N}\left(\sum_{i=0}^{\infty} \lambda^{i} u_{i}\right)\right]_{\lambda=0}, \quad n \geq 0 .
$$

Substituting decomposition series (12) into both sides of (11) gives the following relationship:

$$
\sum_{n=0}^{\infty} u_{n}=\Phi-\mathscr{L}^{-1} \mathscr{R} \sum_{n=0}^{\infty} u_{n}-\mathscr{L}^{-1} \sum_{n=0}^{\infty} A_{n}
$$

From the above equation, the standard Adomian decomposition method defines the components $u_{n}$ by the following recursive relationship:

$$
\begin{aligned}
& u_{0}=\Phi, \\
& u_{n}=-\mathscr{L}^{-1}\left[\mathscr{R} u_{n-1}+A_{n-1}\right], \quad n=1,2, \ldots
\end{aligned}
$$

Further, [15] introduced the convergence-control parameter $\omega(|\omega|<1)$ and new components are constructed, defined by

$$
\begin{aligned}
\widetilde{u}_{n} & =\sum_{k=0}^{n} C_{n}^{k} \omega^{n-k}(1-\omega)^{k+1} u_{k}, \\
C_{n}^{k} & =\frac{n !}{k !(n-k) !},
\end{aligned}
$$

$$
n=0,1, \ldots
$$

A solution of (10) is an infinite series with the computable components $\tilde{u}_{n}$ which contains a new parameter $\omega$; namely,

$$
\bar{u}=\sum_{n=0}^{\infty} \widetilde{u}_{n}
$$

Comparing (15) with (16), it is knowable that the standard Adomian decomposition method is a case of the enhanced technique. The existence of the parameter $\omega$ can adjust the convergence region and it is beneficial to improve the accuracy of the truncated series. It is can be identified by the analysis presented in [22-24], where Professor Liao gives the efficient way to obtain the optimal convergence-control parameters.

3.2. Derivation of the Solution. The finite difference method has been extended successfully to deal with various fractional differential equations [25-27]. Under the technique, the differential equation is transformed into a difference equation and numerical solutions are finally obtained.

The space variable $S$ is discretized by the difference approximation, where $S \in\left[0, S_{\max }\right]$ and $S_{\max }$ is a realistic and practical approximation to infinity. Let the step $h=S_{\max } / M$ and $S_{m}=m h(m=0,1, \ldots, M) ; V^{m}(\tau)$ denotes a solution of (6) in the space point $S_{m}$.
For spatial derivative, the following difference approximations are used:

$$
\begin{aligned}
\frac{\partial V}{\partial S} & =\frac{V^{m+1}-V^{m-1}}{2 h}+O(h), \\
\frac{\partial^{2} V}{\partial S^{2}} & =\frac{V^{m+1}-2 V^{m}+V^{m-1}}{h^{2}}+O\left(h^{2}\right) .
\end{aligned}
$$

Substituting (18) into (6) gives

$$
\begin{aligned}
(m h)^{d_{f}-1} & \frac{\partial^{\alpha} V^{m}(\tau)}{\partial \tau^{\alpha}} \\
= & \frac{1}{2} \sigma^{2} m^{2}\left(V^{m+1}(\tau)-2 V^{m}(\tau)+V^{m-1}(\tau)\right) \\
& +\frac{r m}{2}\left(V^{m+1}(\tau)-V^{m-1}(\tau)\right)-r V^{m}(\tau) .
\end{aligned}
$$

Equation (8) is discretized and gives

$$
\begin{aligned}
V^{m}(0) & =\max (m h-K, 0), \quad m=0, \ldots, M, \\
V^{0}(\tau) & =0, \\
V^{M}(\tau) & =M h-K \exp (-r \tau) .
\end{aligned}
$$

From (9), one can get

$$
\begin{aligned}
V^{m}(0) & =\max (K-m h, 0), \quad m=0, \ldots, M, \\
V^{0}(\tau) & =K \exp (-r \tau), \\
V^{M}(\tau) & =0
\end{aligned}
$$

According to Adomian decomposition method, the linear operator is chosen and the treatment of nonlinear term is not involved for this problem. Equation (19) can be rewritten as

$$
\begin{aligned}
& (m h)^{d_{f}-1} \mathscr{L} V_{k}^{m}(\tau) \\
& =\frac{1}{2} \sigma^{2} m^{2}\left(V_{k-1}^{m+1}(\tau)-2 V_{k-1}^{m}(\tau)+V_{k-1}^{m-1}(\tau)\right) \\
& \quad+\frac{r m}{2}\left(V_{k-1}^{m+1}(\tau)-V_{k-1}^{m-1}(\tau)\right)-r V_{k-1}^{m}(\tau) .
\end{aligned}
$$

The inverse operator of $\mathscr{L}$ is assumed to be a RiemannLiouville fractional integral operator, given by

$$
\mathscr{L}^{-1}(\cdot)=J^{\alpha}(\cdot)=\frac{1}{\Gamma(\alpha)} \int_{0}^{\tau}\left(\tau-\tau^{\prime}\right)^{\alpha-1}(\cdot) d \tau^{\prime} .
$$

Then, the recursive relationship of the call option is defined by

$$
\begin{aligned}
& V_{0}^{m}(\tau)=\max (m h-K, 0), \quad m=0 \cdots M, \\
& V_{k}^{0}(\tau)=0
\end{aligned}
$$




$$
\begin{aligned}
& V_{k}^{m}(\tau)=(m h)^{1-d_{f}} \\
& \cdot J^{\alpha}\left\{\frac{1}{2} \sigma^{2} m^{2}\left(V_{k-1}^{m+1}(\tau)-2 V_{k-1}^{m}(\tau)+V_{k-1}^{m-1}(\tau)\right)\right. \\
& \left.+\frac{r m}{2}\left(V_{k-1}^{m+1}(\tau)-V_{k-1}^{m-1}(\tau)\right)-r V_{k-1}^{m}(\tau)\right\}, \\
& \quad m=1 \cdots M-1, \\
& V_{k}^{M}(\tau)=-K \frac{(-r \tau)^{k}}{k !}, \quad k=1,2, \ldots .
\end{aligned}
$$

For the put option, we have

$$
\begin{aligned}
& V_{0}^{m}(\tau)=\max (K-m h, 0), \quad m=0 \cdots M, \\
& V_{k}^{0}(\tau)=K \frac{(-r \tau)^{k}}{k !}, \\
& V_{k}^{m}(\tau)=(m h)^{1-d_{f}} \\
& \cdot J^{\alpha}\left\{\frac{1}{2} \sigma^{2} m^{2}\left(V_{k-1}^{m+1}(\tau)-2 V_{k-1}^{m}(\tau)+V_{k-1}^{m-1}(\tau)\right)\right. \\
&\left.+\frac{r m}{2}\left(V_{k-1}^{m+1}(\tau)-V_{k-1}^{m-1}(\tau)\right)-r V_{k-1}^{m}(\tau)\right\}, \\
& \quad m=1 \cdots M-1,
\end{aligned}
$$

$V_{k}^{M}(\tau)=0, \quad k=1,2, \ldots$

The components $\widetilde{V}_{n}^{m}$ are determined by the following expressions:

$$
\begin{aligned}
& \widetilde{V}_{n}^{0}(\tau)=V_{n}^{0}(\tau), \\
& \widetilde{V}_{n}^{m}(\tau)=\sum_{k=0}^{n} C_{n}^{k} \omega^{n-k}(1-\omega)^{k+1} V_{k}^{m}(\tau), \\
& \quad m=1 \cdots M-1, \\
& \widetilde{V}_{n}^{M}(\tau)=V_{n}^{M}(\tau), \quad n=0,1,2, \ldots .
\end{aligned}
$$

Eventually, the semianalytical approximate solution of the fractional model (6) is

$$
\bar{V}^{m}(\tau)=\sum_{n=0}^{\infty} \widetilde{V}_{n}^{m}(\tau), \quad m=0 \cdots M
$$

We can also establish other modes according to the recurrence formulas presented by [28]. But, the focus of the work is to explain the application of the time-fractional model, so we do not consider other forms. Further, the initial and boundary conditions that are satisfied by series solution (27) are checked out. For the boundary condition, it is evident via Maclaurin series expansion of $M h-K \exp (-r \tau)$ and $K \exp (-r \tau)$. The following equality tells us that the initial condition can be proved under condition (23). Therefore, series solution (27) is reasonably structured.

$$
\begin{aligned}
\bar{V}^{m}(0) & =\sum_{n=0}^{\infty} \widetilde{V}_{n}^{m}(0) \\
& =\left(1+\omega+\omega^{2}+\cdots+\omega^{n}+\cdots\right) \widetilde{V}_{0}^{m} \\
& =\frac{1}{(1-\omega)}(1-\omega) V_{0}^{m}=V_{0}^{m}, \\
& m=1 \cdots M-1 .
\end{aligned}
$$

\section{Application and Analysis}

With the help of the mathematical calculation software $M A T L A B$, the result of Section 3 is tested in China's financial market.

Example 1. The call warrant of Angang Steel 030001 is taken as an example to explain the application of results (27).

The data (the data came from the database of the Tai'an CSMAR series) from 12/05/2005 to 10/25/2006 are used to estimate parameters. The first step is to determine the parameter $d_{f}$ of the fractional derivative model. Theoretically speaking, it is a Hausdorff dimension. For the self-affine processes, [29] pointed out the celebrated relationship $D+$ $H=n+1$ for a self-affine surface in $n$-dimensional space, where $D$ is a fractal dimension and $H$ is Hurst coefficient. For this problem, Mandelbrot gave some discussions in [29], where he arranged a lot of his own and his partners work. Hurst is a statistic that characterizes the correlation effect of time series. For a time series, the fractal dimension $D$ can be considered as $2-\mathrm{H}$. In practical application, the calculation results are generally approximate. So, the traditional R/S analysis $[30,31]$ is employed to give Hurst exponent. The formula $\ln \left(V_{i+1} / V_{i}\right)$ is applied to calculate the logarithmic return rate of the call warrant, where $V_{i}$ is the daily closing price. Hurst exponent for option is estimated as 0.6207 . The result is greater than 0.5 and proves that the warrants market has the fractal character. This conclusion basically tallies with those studies $[5,6]$ on Chinese financial market.

Secondly, $\sigma$ is estimated by the historical volatility and then is annualized. The formula is

$$
\begin{aligned}
X_{i} & =\ln \left(\frac{S_{i+1}}{S_{i}}\right), \\
\bar{X} & =\frac{1}{N} \sum X_{i}, \\
\sigma & =\sqrt{\frac{242 \sum\left(X_{i}-\bar{X}\right)^{2}}{N-1}},
\end{aligned}
$$

where $S_{i}$ stands for the daily closing price of the stock of Angang Steel and the value 242 is chosen as a general number of trading days. Then the volatilities are 0.3948 . Hurst exponent is 0.6006 and $d_{f}$ takes 1.3994 .

The risk-free interest rate $r$ takes 0.0252 (the data came from the people's bank of China) that is the rate for one-year 


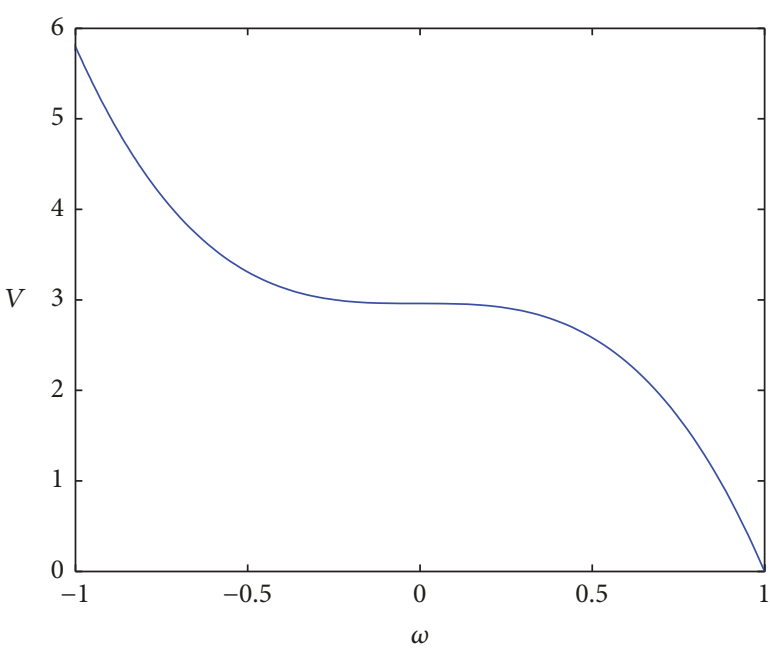

(a) $\alpha=0.3793$

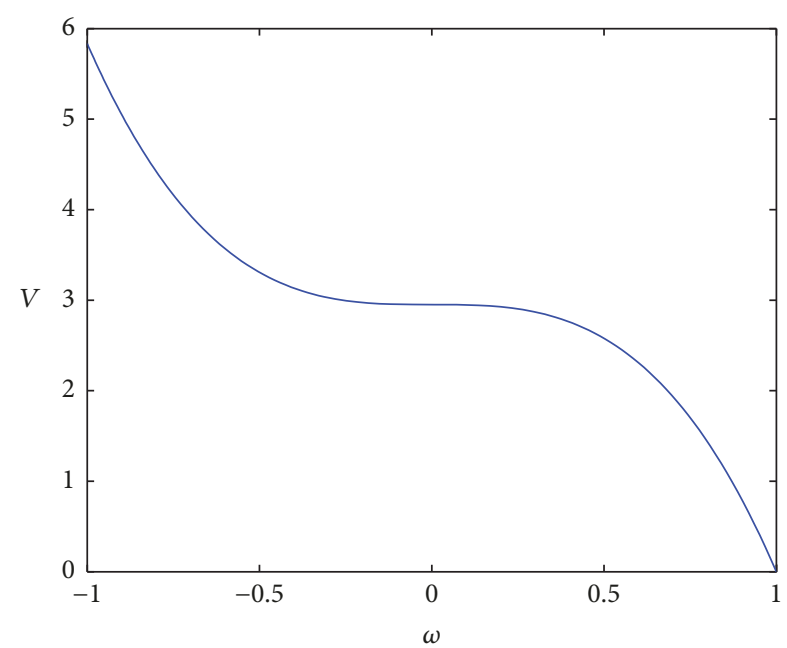

(b) $\alpha=0.6207$

Figure 1: The $\omega$-curve.

term deposit after adjustment in 2006. The strike price $K$ is 3.6. The space length $S_{\max }$ and the step length $h$ are 9 and 0.01 , respectively.

After completing the above parameters estimation, the data $S=6.32, \tau=T-t=24 / 242$, and $V=3.057$ on 10/25/2006 are substituted into the second approximation $\bar{V}_{2}(\tau)=\sum_{n=0}^{2} \widetilde{V}_{n}(\tau)$. Let $\alpha$ be $0.3793(1-\mathrm{H})$, the parameter $\omega$ is solved as -0.4984 and $0.2378 \pm 0.4249$ i. If $\alpha$ is 0.6207 (Hurst exponent), the corresponding $\omega$ is -0.4985 and $0.2428 \pm$ $0.4279 i$.

The next step is to calculate the option price on $10 / 26 / 2006$, when $S=6.54, \tau=T-t=23 / 242$. Based on the convergence-control parameter pointed out by [22-24], the $\omega$-curves of the solution $\bar{V}_{2}^{654}(\tau)$ with $\alpha$ being 0.3793 and 0.6207 are drawn in Figure 1. The interval where the solution curves are near horizontal is valid and the homologous solutions can be convergent. Although Figure 1 gives the curves for the real values of $\omega$, it is knowable that the two sets of values for $\omega$ are acceptable.

Finally, substituting $\tau=T-t=23 / 242$ and the first set of values for $\omega$ into $\bar{V}_{2}^{654}(\tau)$, the obtained warrant values in turn are 3.304 and $3.302 \pm 0.001384 i$ under the condition that $\alpha$ is 0.3793 . Similarly, one can get 3.304 and $3.303 \pm 0.0008738 i$ for $\alpha$ being 0.6207 . The classical Black-Scholes solution is 2.9486. The actual value is 3.256. Enlarging the analyzing scope, the estimations by the paper algorithm, the BlackScholes model, and the actual values of the call warrant on 09/18/2006-11/27/2016 are listed in Figures 2 and 3 under the determined parameters.

The solid line and the diamonds in Figures 2 and 3 are obtained under enhanced technique (26). Actually, our results are complex-valued. The real parts of solutions are cut out and described by the solid line and the diamonds. $\omega$ is one of important parameters in the algorithm and it is determined by the actual values of the previous trading day. That is to say, the function of $\omega$ is not only a control parameter of the series solution, but also a reference point for the fluctuation

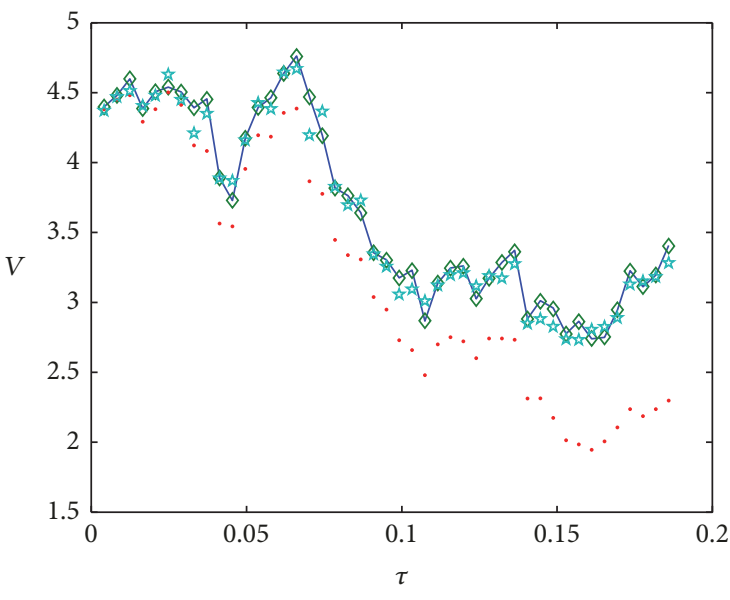

Solid line (Diamond): Real part of solution Dot: Black-Scholes model Star: Actual value

FIgURE 2: $\alpha=0.3793$.

of the option price on the considered trading day. From the concrete example, the valuations by substituting these $\omega$ into the expressions are close. From Figures 2 and 3, we can know that the solid line and the diamonds are very intimate. The trajectory motions of the two curves are along the actual values which are labeled with stars and came from the actual market. In contrast, the dots that describe the values from the classical Black-Scholes model are far from the true values. It is evident that the estimates determined by results (27) are dependable.

Example 2. Considering the SSE 50 ETF put option 10000188 and the call option 10000615 as the second example, the daily closing prices (the data came from the trading software of Essence Securities) of options on 04/23/2015-12/16/2015 and 


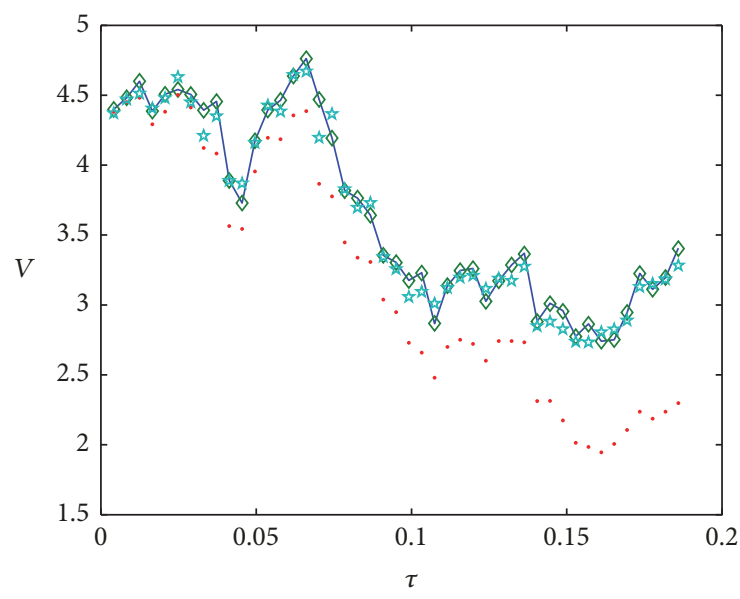

Solid line (Diamond): Real part of solution Dot: Black-Scholes model Star: Actual value

FIGURE 3: $\alpha=0.6207$.

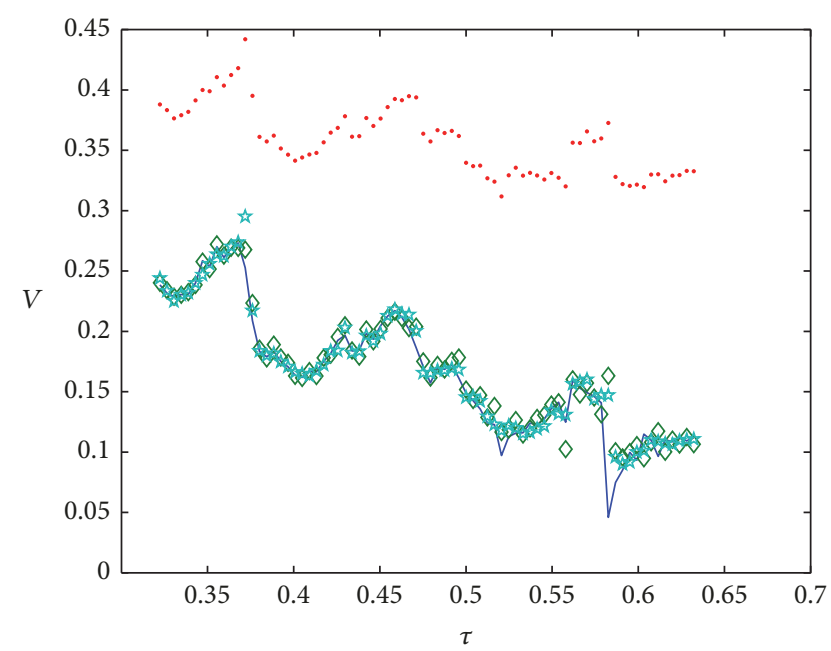

Solid line (Diamond): Real part of solution Dot: Black-Scholes model Star: Actual value

(a) $05 / 13 / 2016-08 / 31 / 2016$

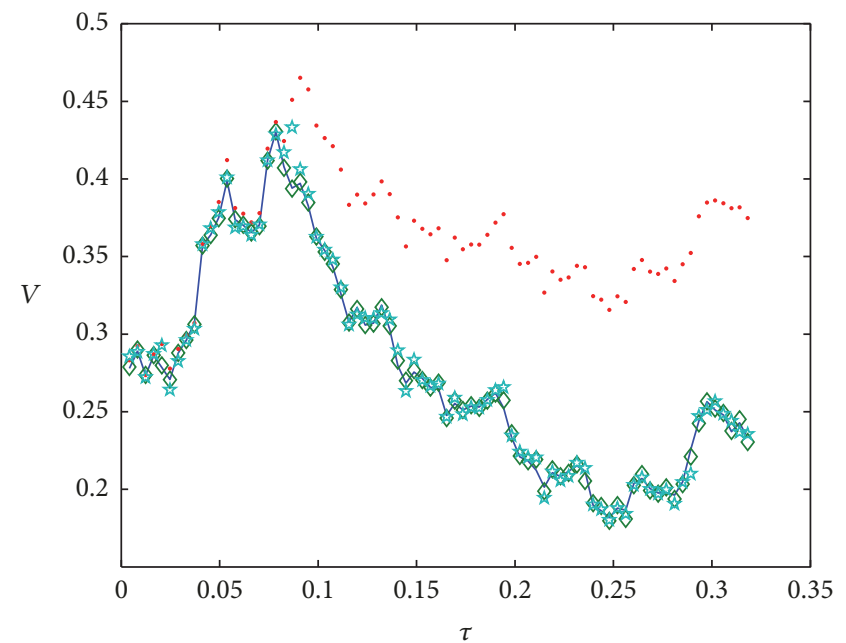

Solid line (Diamond): Real part of solution Dot: Black-Scholes model Star: Actual value

(b) $09 / 01 / 2016-12 / 27 / 2016$

FIGURE 4: $\alpha=0.3433$.

\section{Problems and Conclusions}

In the work, the approximate semianalytical solution of the fractional option pricing model is derived by the enhanced technique and the finite difference method. Further, the result is introduced into the real market. By the two examples in Section 4, one can find that the application of the fractional derivative model in the actual financial market is reliable and valuable. But the existing problems should be pointed out.

(i) The first problem is the parameter estimations. Except for some conventional parameters, the fractional derivative model adds the fractal dimension $d_{f}$ and the order $\alpha$ of fractional derivative. The two parameters make the 


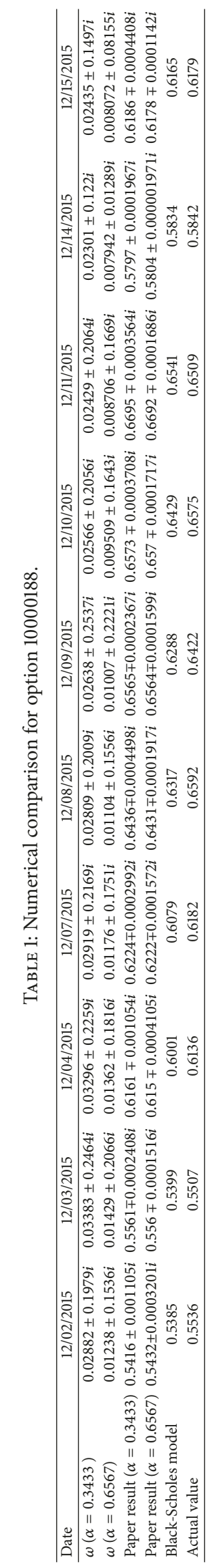




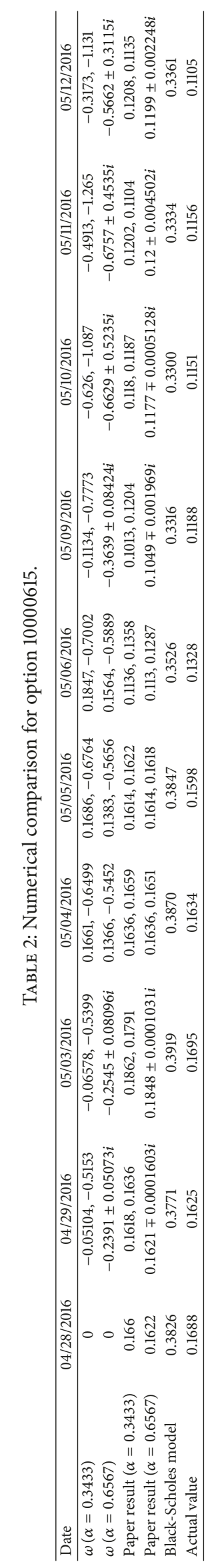




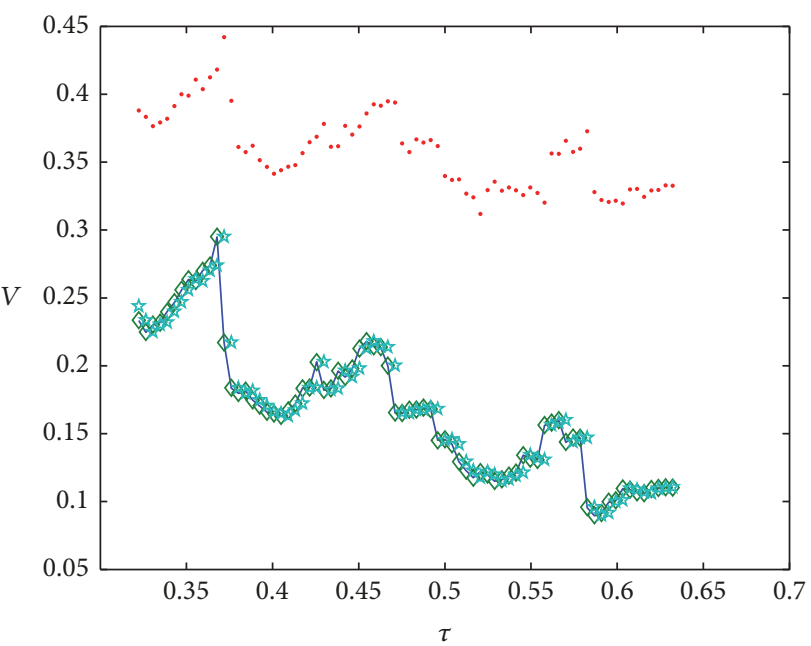

Solid line (Diamond): Real part of solution Dot: Black-Scholes model Star: Actual value

(a) $05 / 13 / 2016-08 / 31 / 2016$

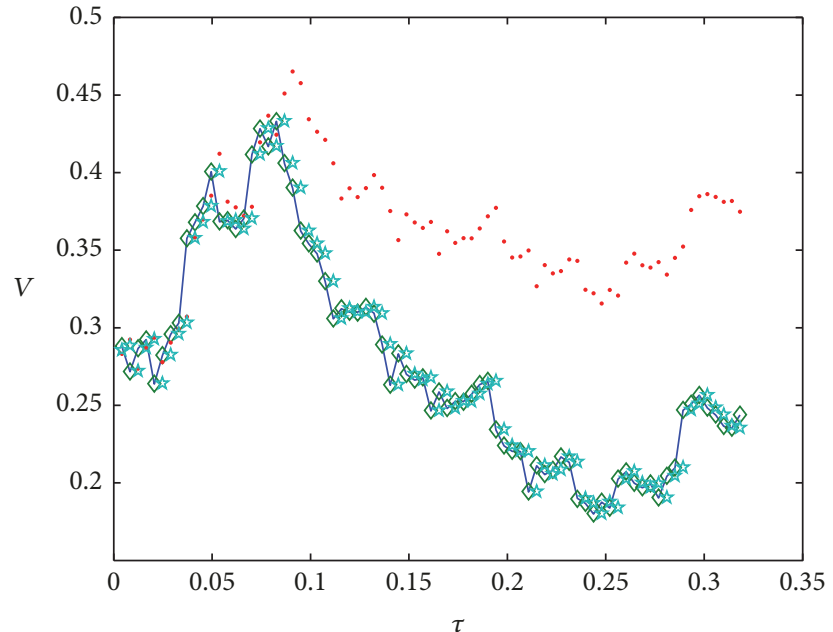

Solid line (Diamond): Real part of solution Dot: Black-Scholes model Star: Actual value

(b) $09 / 01 / 2016-12 / 27 / 2016$

FIGURE 5: $\alpha=0.6567$.

model better display the fractal features of the market. The semianalytical approximate solution presented in this work contains a convergence-control parameter $\omega$ that can improve the accuracy of the approximate solution. However, the existence of these parameters increases the difficulties of the empirical analysis. In the work, $d_{f}$ equals $2-\mathrm{H}$. The relationship between $\omega$ and $\alpha$ on the previous day is used to calculate option price on that day. These are not accurate. If these parameters are made more accurate, one can obtain much better valuation. Their optimal determinations are the key to settle the problems.

(ii) The second problem is the computational difficulties and the actual applications are limited. Example 2 tells us that even the first approximation is also effective. But the point influences judging the rational values of $\omega$ by the $\omega$-curve. If $\omega=0$, the enhanced technique is reduced to the standard Adomian decomposition method. Research experience illustrates that the values of $\omega$ lie in the range close to zero and can be taken as the reasonable estimations. But it is undeniable that the computational difficulties cause the limitations in real application.

Although the fractional partial differential equation has been introduced into the financial theory, the literatures on the practical application are very few. This work makes every effort to apply the fractional partial differential equation to model the actual market. For existing problems, we continue the study in further work. The connections between the fractal dimension, the order of fractional derivative, and Hurst exponent are worthy of studying. In short, the effectiveness and advantage of the fractional derivative model are indubitable, but more problems need further researches. We hope and believe that the study of the fractional derivative model in the financial theory can advance the development of mathematics and finance.

\section{Conflicts of Interest}

The author declares that there are no conflicts of interest regarding the publication of this paper.

\section{Acknowledgments}

The work is supported by the National Natural Science Foundation of China (no. 71501031) and the Project of the Educational Department of Liaoning Province, China (no. LN2017FW010).

\section{References}

[1] F. Black and M. Scholes, "The pricing of options and corporate liabilities," Journal of Political Economy, vol. 81, no. 3, pp. 637654, 1973.

[2] R. C. Merton, "Theory of rational option pricing," Bell Journal of Economics and Management Science, vol. 4, no. 1, pp. 141-183, 1973.

[3] B. Mandelbrot, "The variation of certain speculative prices," The Journal of Business, vol. 36, no. 4, pp. 394-413, 1963.

[4] E. E. Peters, "Fractal structure in the capital market," Financial Analyst, vol. 7, pp. 434-453, 1989.

[5] H. Q. Li and C. Q. Ma, "An empirical study of long-term memory of return and volatility in chinese stock markrt," Journal of Finance and Economics, vol. 31, no. 8, pp. 29-37, 2005.

[6] T. F. Huang, B. Y. Li, and J. X. Xiong, "Test on the chaotic characteristic of chinese futures market," Systems Engineering, vol. 30, no.1, pp. 43-53, 2012.

[7] Á. Cartea and D. del-Castillo-Negrete, "Fractional diffusion models of option prices in markets with jumps," Physica A: Statistical Mechanics and its Applications, vol. 374, no. 2, pp. 749763, 2007. 
[8] O. Marom and E. Momoniat, "A comparison of numerical solutions of fractional diffusion models in finance," Nonlinear Analysis: Real World Applications, vol. 10, no. 6, pp. 3435-3442, 2009.

[9] J. Xi and J. W. Cao, "Fractional partial differential equations and numerical discretization method for pricing american option," Journal on Numerical Methods and Computer Applications, vol. 35, no. 3, pp. 229-240, 2014.

[10] W. Chen, X. Xu, and S.-P. Zhu, "Analytically pricing Europeanstyle options under the modified Black-Scholes equation with a spatial-fractional derivative," Quarterly of Applied Mathematics, vol. 72, no. 3, pp. 597-611, 2014.

[11] G. Jumarie, "Stock exchange fractional dynamics defined as fractional exponential growth driven by (usual) Gaussian white noise. Application to fractional Black-Scholes equations," Insurance: Mathematics and Economics, vol. 42, no. 1, pp. 271-287, 2008.

[12] G. Jumarie, "Derivation and solutions of some fractional BlackScholes equations in coarse-grained space and time: application to Merton's optimal portfolio," Computers \& Mathematics with Applications, vol. 59, no. 3, pp. 1142-1164, 2010.

[13] J.-R. Liang, J. Wang, W.-J. Zhang, W.-Y. Qiu, and F.-Y. Ren, "Option pricing of a bi-fractional Black-Merton-Scholes model with the Hurst exponent $\mathrm{H}$ in $[1 / 2,1]$," Applied Mathematics Letters, vol. 23, no. 8, pp. 859-863, 2010.

[14] W. Chen, X. Xu, and S.-P. Zhu, "Analytically pricing double barrier options based on a time-fractional Black-Scholes equation," Computers \& Mathematics with Applications, vol. 69, no. 12, pp. 1407-1419, 2015.

[15] L. Song, "A space-time fractional derivative model for European option pricing with transaction costs in fractal market," Chaos, Solitons and Fractals, vol. 103, pp. 123-130, 2017.

[16] M. Giona and H. E. Roman, "Fractional diffusion equation on fractals: one-dimensional case and asymptotic behaviour," Journal of Physics A: Mathematical and General, vol. 25, no. 8, pp. 2093-2105, 1992.

[17] M. Giona and H. E. Roman, "Fractional diffusion equation for transport phenomena in random media," Physica A: Statistical Mechanics and Its Applications, vol. 185, no. 1-4, pp. 87-97, 1992.

[18] F.-Y. Ren, Z.-G. Yu, and F. Su, "Fractional integral associated to the self-similar set or the generalized self-similar set and its physical interpretation," Physics Letters A, vol. 219, no. 1-2, pp. 59-68, 1996.

[19] W. Y. Li, Application and numerical solution of fractional order equation in financial model [M.S. thesis], Hangzhou Dianzi University, Hangzhou, China, 2009.

[20] G. Adomian, "A review of the decomposition method in applied mathematics," Journal of Mathematical Analysis and Applications, vol. 135, no. 2, pp. 501-544, 1988.

[21] G. Adomian, Solving frontier problems of physics: the decomposition method, Kluwer Academic Publishers, Massachusetts, Mass, USA, 1994.

[22] S. J. Liao, beyond Perturbation: Introduction to the Homotopy Analysis Method, Chapman and Hall/CRC Press, Boca Raton, Fla, USA, 2003.

[23] S. Liao, "Notes on the homotopy analysis method: some definitions and theorems," Communications in Nonlinear Science and Numerical Simulation, vol. 14, no. 4, pp. 983-997, 2009.

[24] S. Liao, "An optimal homotopy-analysis approach for strongly nonlinear differential equations," Communications in Nonlinear Science and Numerical Simulation, vol. 15, no. 8, pp. 2003-2016, 2010.
[25] S. Chen, F. Liu, and V. Anh, "A novel implicit finite difference method for the one-dimensional fractional percolation equation," Numerical Algorithms, vol. 56, no. 4, pp. 517-535, 2011.

[26] E. Sousa, "A second order explicit finite difference method for the fractional advection diffusion equation," Computers \& Mathematics with Applications, vol. 64, no. 1, pp. 3141-3152, 2012.

[27] Q. Zhang, M. Ran, and D. Xu, "Analysis of the compact difference scheme for the semilinear fractional partial differential equation with time delay," Applicable Analysis: An International Journal, vol. 96, no. 11, pp. 1867-1884, 2017.

[28] L. Song and W. Wang, "A new improved Adomian decomposition method and its application to fractional differential equations," Applied Mathematical Modelling, vol. 37, no. 3, pp. 1590-1598, 2013.

[29] B. B. Mandelbrot, The Fractal Geometry of Nature, W. H. Freeman and Company, New York, NY, USA, 1982.

[30] B. B. Mandelbrot, "Statistical methdology for nonperiodic cycles: from the covariance to R/S analysis," National Bureau of Economic Research, vol. 1, no. 3, pp. 259-290, 1972.

[31] H. E. Hurst, "The long-term storage capacity of reservoirs," Transcactions of the American Society of Civil Engineers, vol. 116, pp. 87-92, 1951. 


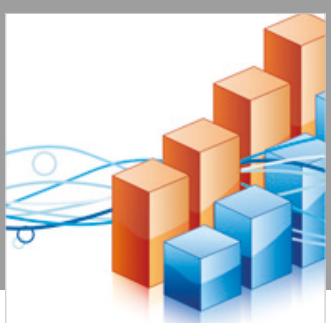

Advances in

Operations Research

\section{-n-m}
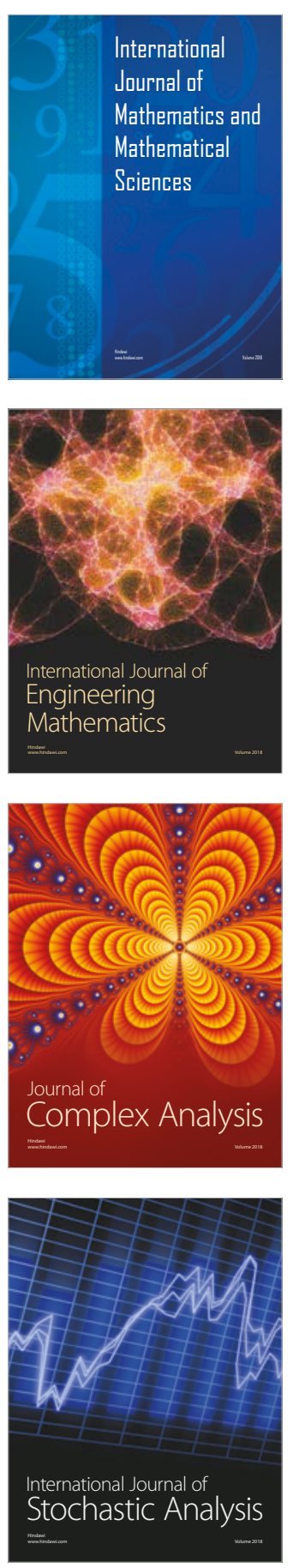
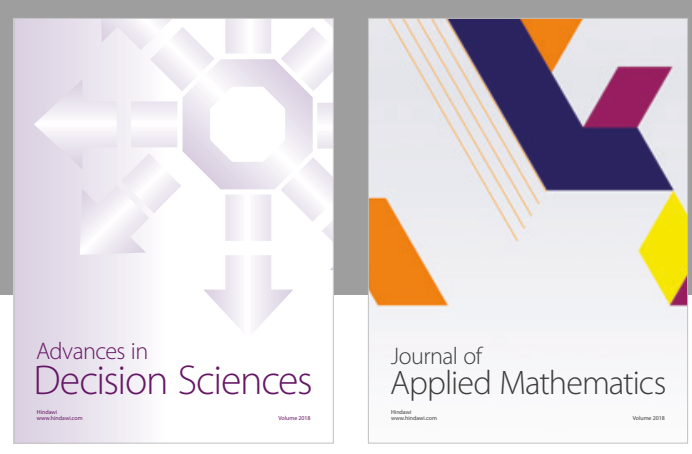

Journal of

Applied Mathematics
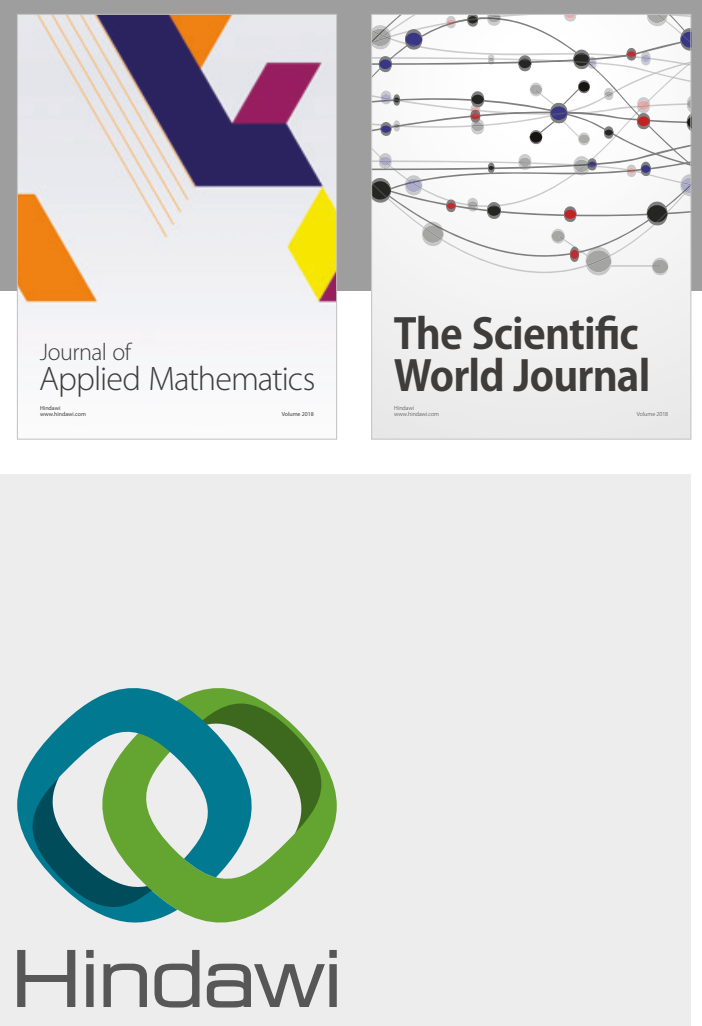

Submit your manuscripts at

www.hindawi.com

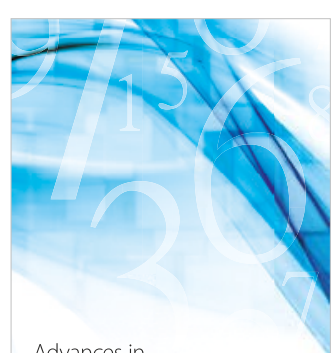

Advances in
Numerical Analysis
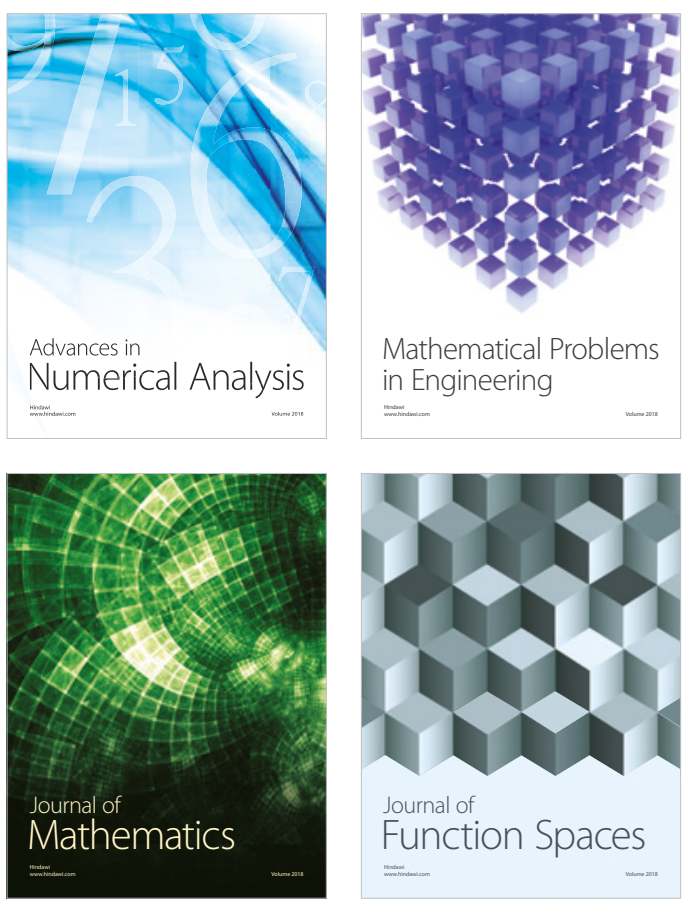

Mathematical Problems in Engineering

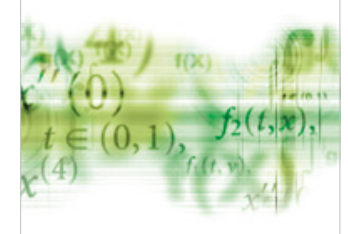

International Journal of

Differential Equations

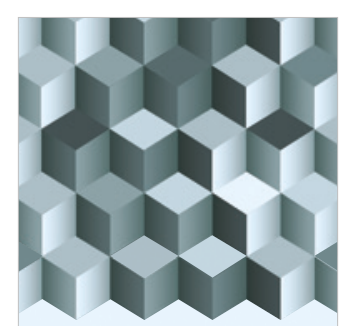

Journal of

Function Spaces
The Scientific

World Journal

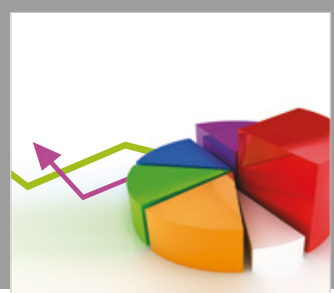

Journal of

Probability and Statistics
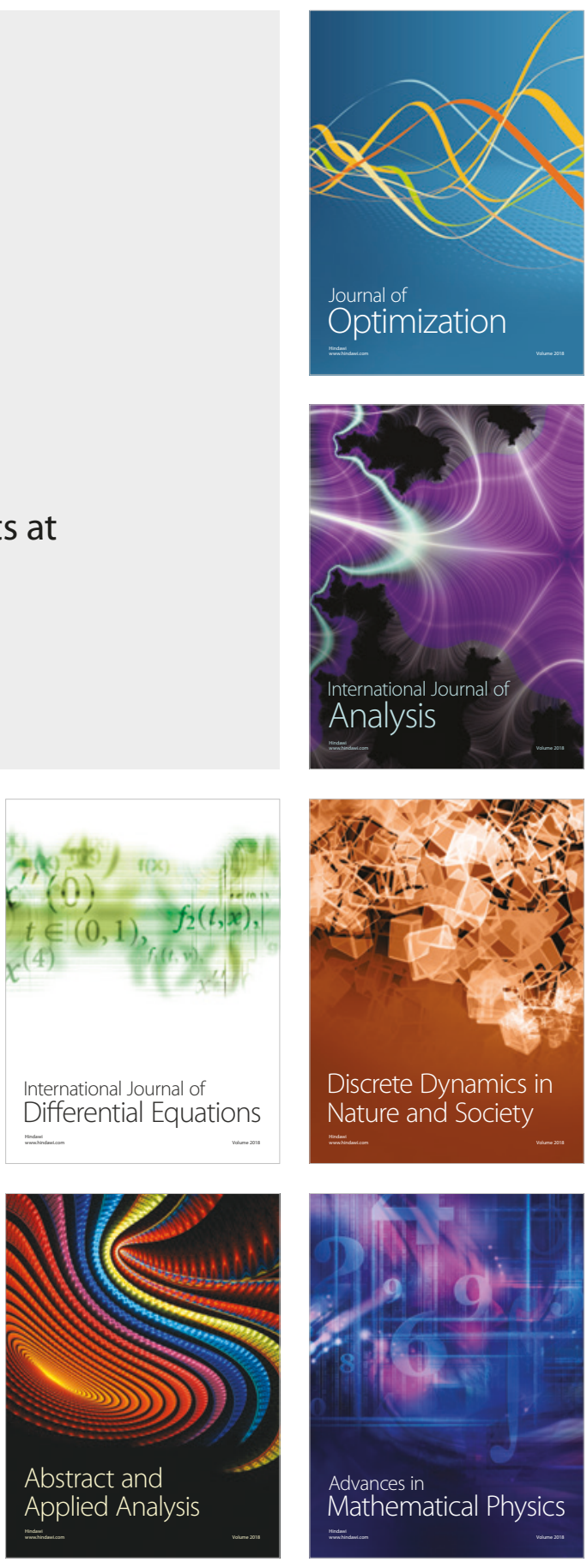